\title{
Calcitonin gene-related peptide regulates FOSL2 expression and cell proliferation of BMSCs via mmu_circRNA_003795
}

\author{
WEN REN* ${ }^{*}$ LAN YANG* ${ }^{*}$ TIAN DENG, CAIJUAN WU, YUANJING LI, \\ JINGWEN WU, ZHU HUANG, FALIANG DU and LVHUA GUO
}

\author{
Key Laboratory of Oral Medicine, Guangzhou Institute of Oral Disease, \\ Stomatology Hospital of Guangzhou Medical University, Guangzhou, Guangdong 510140, P.R. China
}

Received December 25, 2017; Accepted March 7, 2019

DOI: $10.3892 / \mathrm{mmr} .2019 .10038$

\begin{abstract}
Circular RNAs (circRNAs) are a class of non-coding RNAs that may have important regulatory potency in various biological processes. However, the role of circRNAs and their potential functions in bone marrow mesenchymal stem cells of mice (BMSCs) are still ambiguous. The current study aims to examine the expression of circRNAs and to investigate their effects on FOS like 2 AP-1 transcription factor subunit (FOSL2) expression following stimulation of BMSCs with calcitonin gene-related peptide (CGRP). RNA generated from BMSCs stimulated with or without CGRP was used in a microarray to detect expression of circRNAs. There were 58 significantly differentially expressed circRNAs following CGRP treatment, with 44 circRNAs downregulated and 14 upregulated. Bioinformatics analysis and regulatory networks were used to identify the potential interactions between circRNAs and microRNAs (miRs). mmu_circRNA_003795 was significantly increased in the CGRP-stimulated BMSCs compared with the blank control. Silencing of mmu_circRNA_003795, significantly increased the expression of mmu_miR-504-3p, whereas FOSL2 expression and cell proliferation were decreased. Furthermore, silencing of mmu_mir-504-3p using an miR inhibitor led to increased FOSL2 expression. Additionally, silencing of mmu_circRNA_003795 using small interfering RNA induced marked alterations in the cell cycle of BMSCs. The results demonstrated that mmu_circRNA_003795 can indirectly regulate FOSL2 expression via sponging of miR-504-3p, resulting in alterations in BMSC proliferation.
\end{abstract}

Correspondence to: Professor Lvhua Guo, Key Laboratory of Oral Medicine, Guangzhou Institute of Oral Disease, Stomatology Hospital of Guangzhou Medical University, Guangzhou, Guangdong 510140, P.R. China

E-mail: 562210919@qq.com

*Contributed equally

Key words: mmu_circRNA_003795, calcitonin gene-related peptide, bone marrow mesenchymal stem cells

\section{Introduction}

Circular RNAs (circRNAs) are an important type of non-coding endogenous RNA molecules involved in regulating gene expression (1). These molecules were first identified in the 1970s in the RNA virus (2). In 1979, Hsu and Coca-Prados used electron microscopy to observe, for the first time, that RNA could be present in the cytoplasm of eukaryotic cells in a cyclic form (3). Due to the restriction of the technical capabilities at that time, circRNA was only considered to be a type of low-abundance RNA molecule formed by the incorrect splicing of exon transcripts. However, with the continuous improvement of science and technology, especially widely used in bioinformatics and RNA sequencing techniques, it hasbeen established that transcripts of a number of exons can be used to form circRNA through non-linear reverse splicing or gene rearrangement (4).

In contrast to traditional linear RNA, circRNAs are a type of non-coding RNA existing with 1-5 exons forming a closed loop structure. With the rapid development of science and technology circRNAs are now known to be extensively expressed in mammals, have conserved sequences and exhibit structural stability (5-8). More and more studies have demonstrated that circRNAs have important roles in the expression of various regulatory genes (9-11). For example, circRNA-forkhead box protein $\mathrm{O} 3$ has been demonstrated to form ternary complexes with P21 and cyclin dependent kinase 2 to regulate cell cycle changes (12). Additionally, hsa_circ-0067934 can promote the proliferation of esophageal squamous cell carcinoma (13). However, the majority of previous studies have indicated that the main function of circRNAs is to act as an microRNA (miRNA/miR) sponge, achieving the effect of controlling genes by inhibiting the activity of miRNAs $(14,15)$. Bone defects and bone shortening caused by trauma, inflammation and surgical treatment of tumors remains a medical problem threatening human health. Notably, in dental clinics, bone defects tend to affect the efficiency of dental implant restoration, which can reduce patients' quality of life; the quality and quantity of bone is a key consideration following implant restoration (16). Gene expression and cellular ultrastructures indicate that bone marrow mesenchymal stem cells (BMSCs) have the potential for self-renewal and multi-directional differentiation (17) into cells of various tissues, including fat, bone and muscle, 
depending on specific internal and external conditions. In addition, BMSCs are crucial for normal bone development and maintenance of bone metabolism (18). Therefore, increasing the proliferation ability of BMSCs may be of great importance for the repair of bone defects.

Calcitonin gene-related peptide (CGRP) is a biologically active polypeptide consisting of 37 amino acids expressed in the nervous system, respiratory system, digestive system, cardiovascular system and skeleton (19). CGRP is currently the most potent endogenous vasodilator peptide that has been identified (20). CGRP is also a type of neuropeptide, with its highest expression level being in peripheral nerves. Studies have revealed that the expression of CGRP in bone marrow cells, osteoblasts and other cells in bone tissue has an important role in bone repair and reconstruction. CGRP can promote the differentiation and proliferation of osteoblasts, enhance the proliferation of BMSCs, promote BMSC differentiation into osteoblasts and inhibit the formation of osteoclasts (21-23). Previous studies have reported that CGRP effectively promotes BMSC proliferation and osteogenic differentiation $(24,25)$. However, whether CGRP regulates cell proliferation and differentiation via circRNAs has not been previously investigated. In the present study, the role of circRNAs in the CGRP-induced proliferation of BMSCs was investigated to determine whether circRNAs are part of the regulatory mechanism involved, aiming to provide a basis for the further improvement of the osteogenic ability of BMSCs.

\section{Materials and methods}

Cell culture and verification of optimum concentration and time point for CGRP treatment of BMSCs. BMSCs were purchased from Cyagen Biosciences Inc., Guangzhou, China (cat. no. MUBMX-01001) and cultured at $37^{\circ} \mathrm{C}$ with $5 \% \mathrm{CO}_{2}$ in Dulbecco's modified Eagle's medium (Gibco; Thermo Fisher Scientific, Inc., Waltham, MA, USA) supplemented with 10\% FBS (Gibco; Thermo Fisher Scientific, Inc.). BMSCs were seeded on 6-well plates culture dishes at density of $1 \times 10^{5}$ cells per well. In the concentration experiments, BMSCs were stimulated with different concentrations $\left(10^{-7}, 10^{-8}, 10^{-9}\right.$, $10^{-10}, 10^{-11}$ or $0 \mathrm{M}$ ) of CGRP (Sigma-Aldrich; Merck KGaA, Darmstadt, Germany) for 1, 3 or 7 days. The cells were then treated with Cell Counting Kit-8 (CCK8; Dojindo, Molecular Technologies, Inc., Kumamoto, Japan) reagent for $2 \mathrm{~h}$ in the dark to assess the BMSCs proliferation rate. The absorbance of the culture media was measured with a microplate reader (Thermo Fisher Scientific, Inc.) at $450 \mathrm{~nm}$.

Microarray hybridization. The BMSCs were divided into 2 groups: The control group and the subject group. In the subject group, the cells $\left(1 \times 10^{5}\right)$ were treated with $10^{-9}$ M CGRP for 3 days. In the control, the cells were untreated. The total and circular RNAs of the 2 groups was isolated using TRIpure (Aidalb Biotechnologies Co., Ltd., Beijing, China). Total RNA and RNA in each group were quantified using NanoDrop 2000 (Thermo Fisher Scientific, Inc., Wilmington, DE, USA). Sample labeling and array hybridization were performed according to the protocol of the manufacturer (Arraystar, Inc., Rockville, MD, USA). Briefly, circRNA was treated with RNase R (Epicentre; Illumina, Inc., San Diego, CA, USA) to remove linear RNA. Each sample was then amplified and transcribed into fluorescent cRNA using the random priming method (Arraystar Super RNA Labeling kit; Arraystar, lnc.). The labeled cRNA was purified using an RNeasy Mini kit (Qiagen, Inc., Valencia, CA, USA). The concentration and specific activity of the labeled cRNA (pmol Cy3/ $\mu \mathrm{g}$ cRNA) were measured using a NanoDrop ND-1000. Each labeled cRNA $(1 \mu \mathrm{g})$ was fragmented by the addition of $5 \mu \mathrm{l} 10 \mathrm{X}$ blocking agent and $1 \mu 125 \mathrm{X}$ fragmented buffer solution. Then, the mixture was heated at $60^{\circ} \mathrm{C}$ for $30 \mathrm{~min}$. Finally, $25 \mu \mathrm{l} 2 \mathrm{X}$ hybridization buffer was added to dilute the labeled cRNA. Hybridization solution $(50 \mu \mathrm{l})$ was dispensed into the gasket slides and assembled onto the circRNA expression microarray slides which were then incubated in an Agilent hybrid box at $65^{\circ} \mathrm{C}$ for $17 \mathrm{~h}$, with the hybrid array washed, scanned by the G2505C Agilent scanner (version 11.0.1.1; Agilent Technologies, Inc., Santa Clara, CA, USA).

Microarray data analysis. Quantile normalization of the original data and subsequent data processing was performed using the $\mathrm{R}$ software package (version 3.1.2) (25). Following quantile normalization of the original data was performed, low-intensity filtering was carried out and the circRNAs with P or M ('all target values') tagged in at least one or two samples were retained for further analysis. When comparing the two sets of contours, the 'fold-change' (namely, the ratio of the group mean) between groups of circRNAs was calculated. The statistical significance of the differences between groups was determined using a t-test. CircRNAs exhibiting a significant difference in expression exhibited a fold-change $>2$ and a P-value $<0.05$. The sorting and filtering function of Microsoft Excel was used to filter data, analyze output and sort the circRNAs with differential expression according to the fold-changes and P-values. In addition, the interaction between circRNAs and miRNAs was predicted using The relationship between circRNAs and miRNAs was predicted by miRNA target prediction software based on TargetScan (http://www.targetscan.org/), miRanda (http://www.microrna. org/), miRDB (http://www.mirdb.org/). All differentially expressed circRNAs were annotated in detail with information on the interaction between the circRNAs and miRNAs. Cytoscape (version 3.6.0; https://cytoscape.org) software was used to generate a network map, of upregulated circRNAs and their corresponding miRNAs.

Reverse transcription-quantitative polymerase chain reaction (PCR) and nucleic acid electrophoresis. Primer premier 5.0 software was used for mmu_circRNA_003795 primer design. The sequence of the designed primer and the sequence of mmu_circRNA_003795 were compared with Blast database (https://blast.ncbi.nlm.nih.gov/) sequences, and the forwards and reverse primer were matched with the sequence of mmu_circRNA_003795. The total RNA of BMSCs was extracted using the TRIzol method (Thermo Fisher Scientific, Inc.). The quantity and quality of RNA were determined using a Nano Drop 2000 and by electrophoresis on a $10 \mathrm{~g} / \mathrm{l}$ agarose gel. Following RNA extraction, SuperScript III reverse transcriptase (Invitrogen; Thermo Fisher Scientific, Inc.) was used to synthesize cDNA according to the manufacturer's protocol. Subsequently, PCR was performed using 12.5 $\mu 1$ SYBR Premix 
EX Taq (Takara Bio, Inc., Otsu, Japan), $0.5 \mu l$ forward primer (10 pmol/l), $0.5 \mu \mathrm{l}$ reverse primer (10 pmol/l; Table I), $1 \mu \mathrm{l}$ cDNA and $\mathrm{ddH}_{2} \mathrm{O}$ was added to a final volume of $25 \mu \mathrm{l}$. The reaction conditions were as follows: $95^{\circ} \mathrm{C}$ for $30 \mathrm{sec}$; and $95^{\circ} \mathrm{C}$ for $5 \mathrm{sec}, 55^{\circ} \mathrm{C}$ for $30 \mathrm{sec}$ and $72^{\circ} \mathrm{C}$ for $30 \mathrm{sec}$ for 40 cycles. The instrument automatically generated the cycle quantification (Cq) value of mRNA and the internal reference gene, GAPDH. $\mathrm{Cq}$ value difference $(\triangle \mathrm{Cq})$ indicated the relative expression of each mRNA investigate (26). DNA samples were separated by electrophoresis at $110 \mathrm{~V}$ for $30 \mathrm{~min}$ and observed using a gel imager.

CircRNA and miRNA interference. The small interfering (si)RNAs targeting mmu_circRNA_003795 were designed and synthesized by Guangzhou RiboBio Co., Ltd. (Guangzhou, China). The sequences of siRNA were as follows: (Sense 5'-GCU AGAACAGCAUGGUCCAdTdT-3' and anti-sense 3'-dTdTCG AUCUUGUCGUACCAGGU-5'). The target sequence corresponding to the sense and antisense siRNA oligonucleotides was as follows: 5'-GCTAGAACAGCATGGTCCA-3'. Negative control siRNA was designed and synthesized by Guangzhou RiboBio Co., Ltd. (Guangzhou, China; the sequence is confidential). The mmu-miR-504-3p inhibitor was synthesized by Invitrogen (Thermo Fisher Scientific, Inc.). The sequence was as follows: 5'-AGGGAGAGCAGGGCAGGGUUUC-3'. BMSCs $\left(1 \times 10^{5}\right)$ were transfected with $20 \mathrm{nM}$ siRNA or inhibitor using GenMute $^{\mathrm{TM}}$ siRNA Transfection kit and Transfection Buffer (SignaGen Laboratories, Rockville, MD, USA) according to the manufacturer's protocol. Then the cells were incubated for $72 \mathrm{~h}$ at $37^{\circ} \mathrm{C}$ prior to the sequential tests.

Western blotting. Protein was extracted using radioimmunoprecipitation assay buffer $[50 \mathrm{mM}$ Tris $\mathrm{HCl}(\mathrm{pH}$ 7.4), $150 \mathrm{mM}$ $\mathrm{NaCl}, 1 \%$ Nonidet P40 and $0.1 \%$ sodium dodecyl sulfate] and phenylmethanesulfonyl fluoride at $4^{\circ} \mathrm{C}$. Bicinchoninic acid protein assay kit (BestBio, Shanghai, China) was used to determine the concentration. Total protein $(10 \mu \mathrm{g}$ per well) was separated using 10\% SDS PAGE and then transferred to a polyvinylidene difluoride membrane. The membranes were blocked using skimmed milk for $1 \mathrm{~h}$ at room temperature, then the blots were incubated with primary antibody against FOS like 2 AP-1 transcription factor subunit (FOSL2; 1:1,000; ab124830; Abcam, Cambridge, UK) overnight at $4^{\circ} \mathrm{C}$. Following washing in $0.1 \%$ Tween PBS-T, the blots were incubated in goat anti-mouse horseradish peroxidase-conjugated secondary antibody (1:5,000; ab6789; Abcam, Cambridge, $\mathrm{UK})$ for $1 \mathrm{~h}$ at room temperature. Proteins were detected using electrochemiluminescence (ECL kit; Beijing Dingguo Changsheng Biotechnology Co., Ltd., Beijing, China). Image J (version k1.45; National Institutes of Health, Bethesda, MD, USA) was used to measure densitometry.

Cell proliferation assay. Cell proliferation was determined using the Cell Counting Kit-8 (CCK8; Dojindo, Molecular Technologies, Inc., Kumamoto, Japan), according to the manufacturer's protocol. Transfected cells were seeded in 96-well plates (3,000 cells per well) with proliferation detected at $24 \mathrm{~h}$ intervals. Briefly, $10 \mu$ l CCK-8 solution was added to each hole and incubated at $37^{\circ} \mathrm{C}$ for $2 \mathrm{~h}$. Absorbance was then measured at $450 \mathrm{~nm}$ was measured using a spectrophotometer.
Flow cytometry analysis. Cells were washed and resuspended in cold PBS and incubated in ice-cold $70 \%$ ethanol for $3 \mathrm{~h}$. The cells were then centrifuged at $845 \mathrm{x} \mathrm{g}$ and $4^{\circ} \mathrm{C}$ for $10 \mathrm{~min}$ and resuspended in PI/RNase Staining Buffer master mix (BD Pharmingen, BD Biosciences, Franklin Lakes, NJ, USA; $40 \mathrm{mg} / \mathrm{ml}$ PI and $100 \mathrm{mg} / \mathrm{ml}$ RNase in PBS) at a density of $5 \times 10^{5}$ cells $/ \mathrm{ml}$ and incubated at $37^{\circ} \mathrm{C}$ for $30 \mathrm{~min}$ prior to flow cytometry analysis by flow cytometry (27). (BD FACSCanto II, BD Biosciences; ModFit LT 3.2, Verity Software House, Inc., Topsham, ME, USA).

Statistical analysis. All data were analyzed using the SPSS 11.5 software package (SPSS, Inc., Chicago, IL, USA). The nominal data, including the genotype and allele frequency were calculated by a direct gene counting method. Genotype and allele frequencies of each group were analyzed using $\chi^{2}$. Additionally, the odds ratio and $95 \%$ of confidence interval were used to indicate the relative risk. Continuous data was expressed as the mean \pm standard deviation and the comparison of the data between groups was performed using t-test or analysis of variance according to the nature of the data. Each experiment was repeated three times. The data of three groups were evaluated using analysis of variance and least significant difference post hoc tests when the variance was normal, otherwise Dunnett's T3 test was used. $\mathrm{P}<0.05$ was considered to indicate a statistically significant difference.

\section{Results}

Verification of optimum concentration of CGRP on cell proliferation of BMSCs. To determine the effect of CGRP on proliferation of BMSCs, cells were stimulated with different concentrations $\left(10^{-7}, 10^{-8}, 10^{-9}, 10^{-10}, 10^{-11}\right.$ or $\left.0 \mathrm{M}\right)$ of CGRP for 1,3 or 7 days. The CCK- 8 assay demonstrated that CGRP stimulated proliferation at $10^{-9} \mathrm{M}$ (Fig. 1).

Microarray data analysis. High-throughput chip data analysis identified a total of 58 differentially expressed circRNAs when comparing the two treatment groups (the cells were treated with $10^{-9} \mathrm{M}$ CGRP for 3 days and the control cells were untreated). The scatter diagram and hierarchical clustering demonstrate that expression of circRNAs of the CGRP-treated BMSCs was different compared with the blank control group (Fig. 2A and B). The differentially expressed circRNAs included 17 exonic, 16 intragenic and 10 intronic-derived RNAs (Fig. 2C). More circRNAs were downregulated than upregulated (44 and 14, respectively). The seed sequences of the circRNA were compared with miRNA sequences to identify the miRNAs that potentially bind with circRNAs (Fig. 2D). Cytoscape software was used to generate a network map, of upregulated circRNAs and their corresponding miRNAs and target genes.

Microarray data analysis, identified the upregulated and downregulated circRNAs, and those with more predicted target area that could combine with miRNA were selected. Therefore, the miRNAs that bind with the differentially expressed circRNA were predicted. miRDB (http://www. mirdb.org/), TargetScan (http://www.targetscan.org/) and mirbases (http://www.mirbase.org/) were used to determine the corresponding target genes of these miRNAs. Target genes 
Table I. The primers of the detected genes.

\begin{tabular}{ll}
\hline Name & \multicolumn{1}{c}{ Primer } \\
\hline FOSL2 & F: 5'-AGCAGGGATGGACAAGAC-3' \\
& R: 5'-TGGGGTAGGTGAAGACAA-3' \\
ALP & F: 5'-GAGGCATACGCCATCACATG-3' \\
& R: 5'-CCGATGGCACACCTGCTT-3' \\
OCN & F:5'-TCTGACAAAGCCTTCATGTCCA-3' \\
& R: 5'-AACGGTGGTGCCATAGAT-3' \\
GAPDH & F: 5'-AAGAAGGTGGTGAAGCAGG-3' \\
OSX & R: 5'-GAAGGTGGAAGAGTGGGAG-3' \\
Funx2 & R: 5'-GCAAATGACTACCCACCCTT-3' \\
Fmu_circRNA_-ACGAGCCATAGGGATGAGTC-3' & F: 5'-CCTTAGCACCTGCCTTCTTG-3' \\
003795 & R: 5'-TTGCTGTTGCTGTTGTTG-3' \\
miR-504-3p & 5'-AGCAGGGCAGGGTTTCAAA-3' \\
U6 & 5'-GCGCGTCGTGAAGCGTTC-3' \\
Inhibitor- & 5'-AGGGAGAGCAGGGCAGGGUUUC-3' \\
miR-504-3p &
\end{tabular}

miR, microRNA; circRNA, circular RNA; ALP, alkaline phosphatase, tissue nonspecific isozyme; OCN, osteocalcin; FOSL2, FOS like 2 AP-1 transcription factor subunit; Runx 2, runt-related transcription factor 2; OSX, Sp7 transcription factor.

with at least two interrelated predictions in the above databases were selected for further investigation. According to the analysis, mmu_circRNA_003795, miR-504-3p and FOSL2 may have an influence on the proliferation and differentiation of BMSCs.

Verification of the expression of mmu_circRNA_003795 in BMSCs. As demonstrated in Fig. 3A, the potential binding sites of mmu_circRNA_003795 and miR-504-3p were predicted using bioinformatics tools. Furthermore, RT-qPCR was used to analyze the expression of osteogenesis-associated genes in cells treated with CGRP and the blank control group. Osteogenic and proliferation associated genes were significantly upregulated by CGRP, as demonstrated by the following: FOSL2, 3.6-fold ( $\mathrm{P}=0.027)$; alkaline phosphatase, tissue nonspecific isozyme (ALP), 4.3-fold times $(\mathrm{P}=0.044)$; osteocalcin $(\mathrm{OCN})$, 3.7-fold ( $\mathrm{P}=0.027)$; runt-related transcription factor 2 (Runx2), 3.6-fold $(\mathrm{P}=0.01)$ and $\mathrm{Sp} 7$ transcription factor $(\mathrm{OSX}), 2.3$-fold (Fig. 3B; $\mathrm{P}=0.017$ ). Primer premier 5 software was used for mmu_circRNA_003795 primer design. As part of the design, it was required that the product should be able to cross the ring formation node. The sequence of the designed primer and the sequence of mmu_circRNA_003795 were compared with Blast database sequences, and the forwards and reverse primer were perfectly matched with the sequence of mmu_circRNA_003795. RT-qPCR was used for validation of the primers. The expression of mmu_circRNA_003795 in the CGRP-stimulated cells was significantly 2.9-fold higher compared with the control

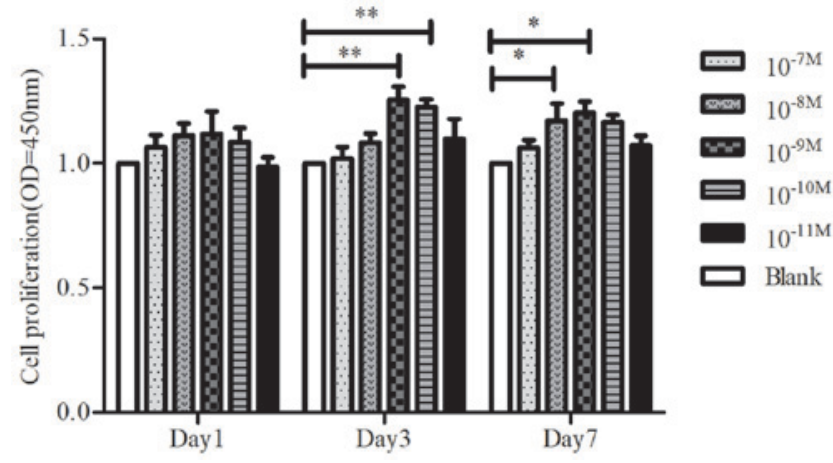

Figure 1. Bone mesenchymal stem cells stimulated with different concentrations $\left(10^{-7}, 10^{-8}, 10^{-9}, 10^{-10}, 10^{-11}\right.$ or $\left.0 \mathrm{M}\right)$ of calcitonin gene-related peptide for 1,3 and 7 days. Cell Counting Kit- 8 was used to assess proliferation. ${ }^{*} \mathrm{P}<0.05$ and ${ }^{* *} \mathrm{P}<0.01$. OD, optical density.

group; the results were in accordance with the microarray data ( $\mathrm{P}=0.004$; Fig. 3C). Additionally, electrophoresis was used to test the PCR product, confirming the upregulated expression of mmu_circRNA_003795 (Fig. 3D). Electrophoresis clearly demonstrated that the PCR product was the expected size and a single band. Furthermore, compared with the blank control group, the mmu_circRNA_003795 expression in the CGRP-stimulated BMSC group was upregulated. The PCR product was sequenced and matched the mmu_circRNA_003795 sequence, including the ring formation node (Fig. 3D). It was demonstrated that the sequence of the product was almost an exact match with the mmu_circRNA_003795 sequence (positive 97\% and negative $100 \%$ ) and contained the ring formation node.

Alterations in the expression of miR504-3p and FOSL2 following mmu_circRNA_003795 interference. The siRNA sequences were designed to silence mmu_circRNA_003795. At $72 \mathrm{~h}$ following transfection, the mmu_circRNA_003795 expression in the siRNA group and negative control group were detected by RT-qPCR. The expression of mmu_ circRNA_003795 in the siRNA group was 0.46-fold decreased compared with the negative control group $(\mathrm{P}=0.005)$, which indicated that si-mmu_circRNA_003795 significantly inhibited the expression of mmu_circRNA_003795 (Fig. 4A). Following successful silencing of mmu_circRNA_003795, the expression of miR-504-3P and FOSL2 was determined by RT-qPCR. The results demonstrated that the expression of miR-504-3P was significantly increased by 6.6-fold $(\mathrm{P}=0.004$; Fig. 4B) and the expression of FOSL2 was significantly decreased by 0.6 -fold $(\mathrm{P}=0.024$; Fig. 4C) following mmu_circRNA_003795 silencing. The western blot analysis demonstrated that silencing of mmu_circRNA_003795 reduced the expression of FOSL2 protein (Fig. 4D and E).

Alterations in the expression of FOSL2 following miR504-3p interference. An miRNA inhibitor sequence was used to block the function of miR-504-3p. At $72 \mathrm{~h}$ following transfection, miR-504-3p expression was detected by RT-qPCR. As demonstrated in Fig. 5A, the miRNA inhibitor significantly decreased the expression of miR-504-3p by 0.363 -fold $(\mathrm{P}=0.005)$. Following the use of an inhibitor to silence miR-504-3p expression, the expression of the FOSL 2 mRNA was also detected. The expression of FOSL2 was significantly increased by 
A

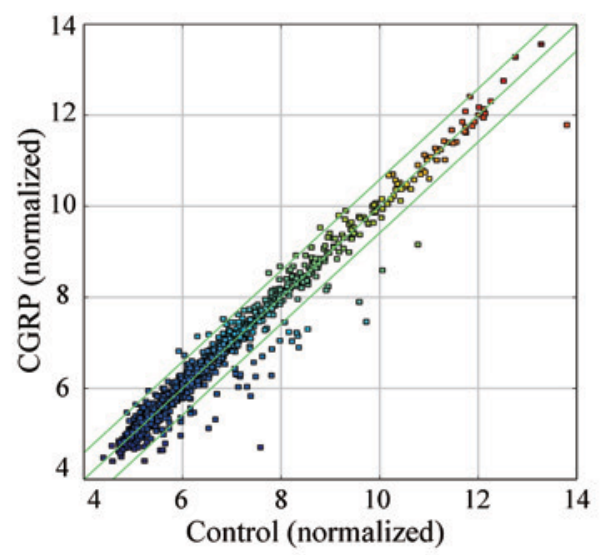

B

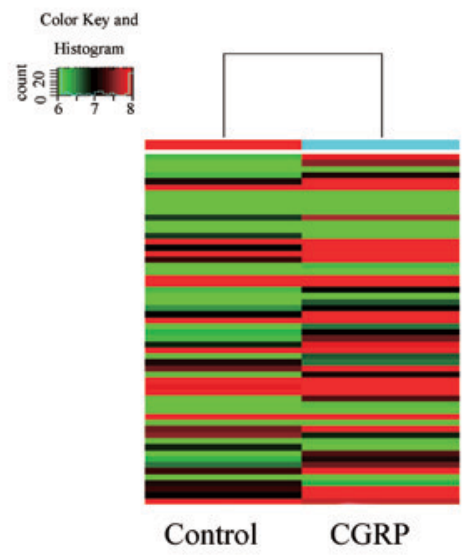

$\mathrm{C}$
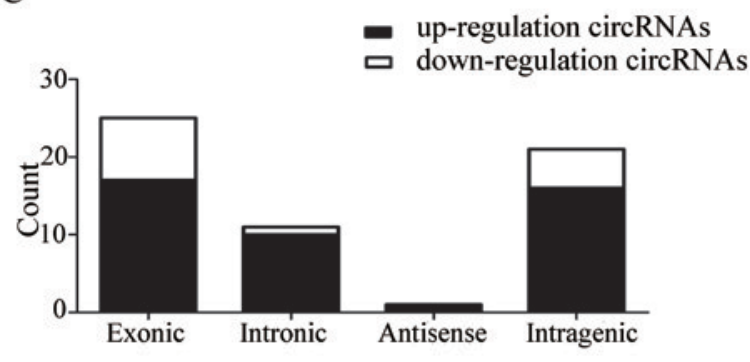

$\mathrm{D}$

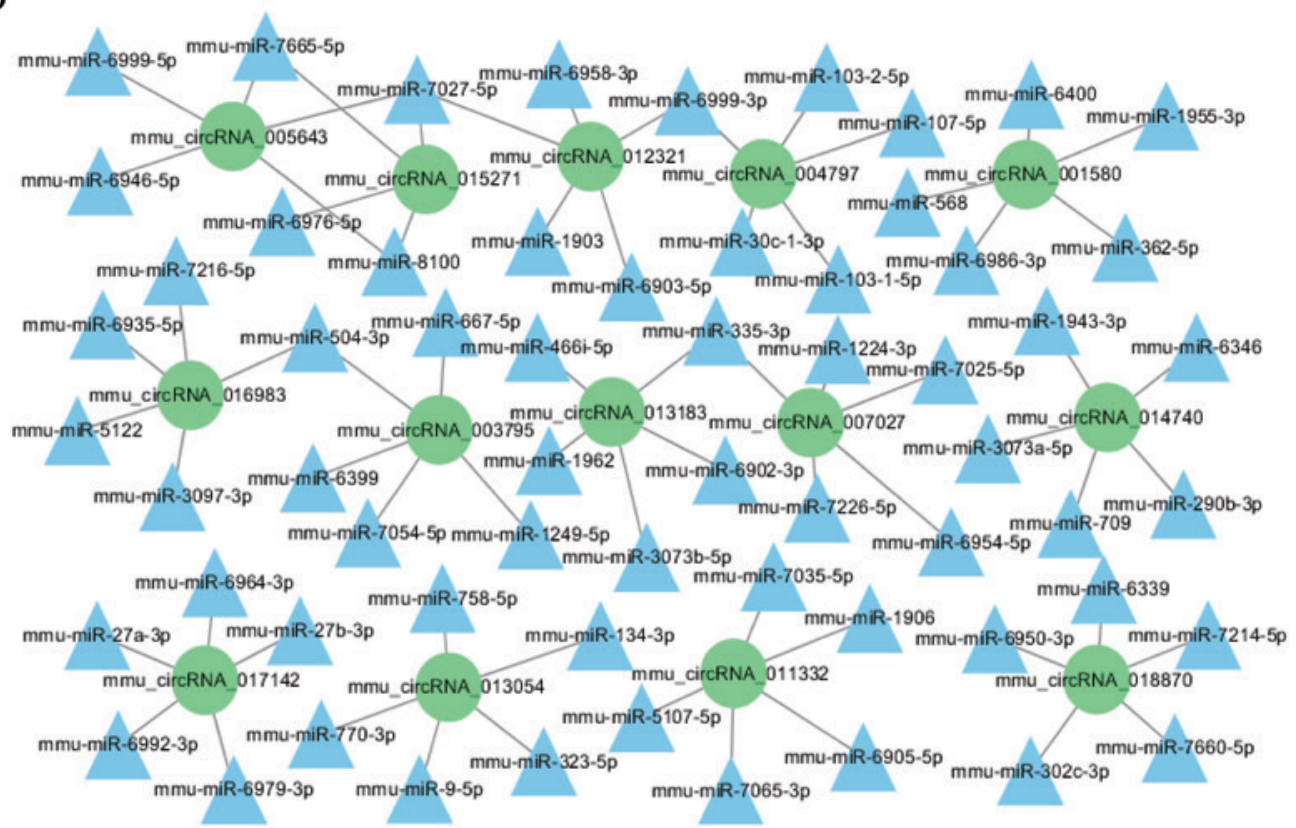

Figure 2. Microarray data analysis. (A) Scatter diagram and (B) hierarchical clustering comparing the expression of circRNAs in the bone mesenchymal stem cell experimental group (stimulated with CGRP) and the blank control group. In (A), red indicates a large change fold, blue indicates a small change fold, and the gradient from blue to red indicates a change in the expression fold of different circRNAs. (C) CircRNAs with differential expression included 17 exonic, 16 intragenic and 10 intronic. Certain circRNAs were from other sources. More circRNAs were downregulated (43) than were upregulated (14) in CGRP-stimulated cells compared with the controls. (D) Cytoscape software created a network map of circRNAs with upregulated expression and their target miRNAs and genes. CGRP, calcitonin gene-related peptide; circRNA, circular RNA; miR, microRNA.

3.3-fold ( $\mathrm{P}=0.044$; Fig. 5B) when the inhibitor of miR-504-3p was used compared with the negative control. Additionally, BMSCs were co-transfected with si-mmu_circRNA_003795 and miR-504-3p inhibitor for $72 \mathrm{~h}$. Compared with the control group, the mRNA expression of FOSL2 was significantly increased by 2.24 -fold ( $\mathrm{P}=0.022$; Fig. $5 \mathrm{~B})$. Western blot analysis demonstrated that inhibition of miR-504-3p increased the expression of FOSL2 protein and expression was also increased in the si-mmu_circRNA_003795 and miR-504-3p inhibitor-g groups (Fig. 5C and D).

mmu_circRNA_003795 promotes cell proliferation and cell cycle changes. The CCK-8 assay was used to detect cell proliferation. The proliferation of BMSCs in the negative 


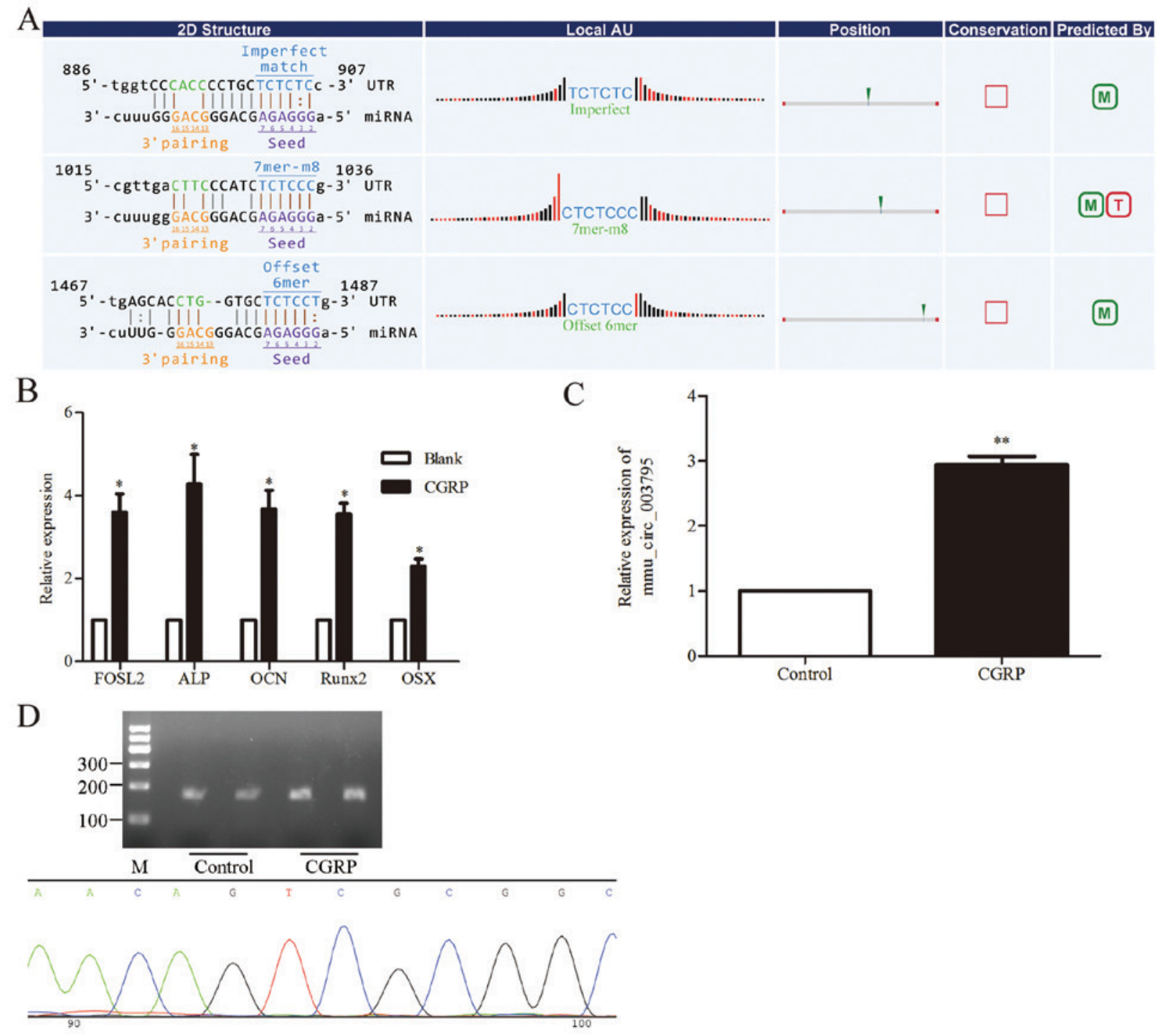

Figure 3. mmu_circRNA_003795 expression in bone mesenchymal stem cells. (A) Potential binding sites of mmu_circRNA_003795 and miR-504-3p were predicted using bioinformatics tools. (B) RT-qPCR analysis of osteogenic genes in CGRP-stimulated cells and the blank control group. (C) RT-qPCR validation of mmu_circRNA_003795 in the CGRP-stimulated group and control group. (D) Agarose electrophoresis of the PCR product demonstrates the expected size and a single band. Sequencing of the product matched mmu_circRNA_003795. ${ }^{*} \mathrm{P}<0.05$ and ${ }^{* * *} \mathrm{P}<0.01$ vs. the control. RT-qPCR, reverse transcriptase-quantitative polymerase chain reaction; CGRP, calcitonin gene-related peptide; circRNA, circular RNA; FOSL2, FOS like 2 AP-1 transcription factor subunit; OSX, Sp7 transcription factor; ALP, alkaline phosphatase; OCN, osteocalcin.

control group was significantly increased compared with the si-mmu_circRNA_003795 group $(24 \mathrm{~h}, \mathrm{P}=0.001$; $48 \mathrm{~h}, \mathrm{P}=0.014 ; 72 \mathrm{~h}, \mathrm{P}=0.042)$, which indicated that $\mathrm{mmu}$ circRNA_003795 promotes the proliferation of BMSCs (Fig. 6A). Additionally, flow cytometry was used to analyze the cell cycle in cells with silenced mmu_circRNA_003795. The si-mmu_circRNA_003795 group exhibited more cells in $\mathrm{G}_{0}-\mathrm{G}_{1}$ phase and less cells in the $\mathrm{G}_{2}-\mathrm{M}$ and $\mathrm{S}$ phase compared with the negative control group $\left(\mathrm{G}_{1}, \mathrm{P}=0.016 ; \mathrm{G}_{2}, \mathrm{P}=0.018 ; \mathrm{S}\right.$, $\mathrm{P}=0.039$; Fig. 6B), indicating that $\mathrm{mmu}$ _circRNA_003795 may regulate the proliferation of BMSCs cells by altering the cells cycles.

\section{Discussion}

circRNAs are circular RNA molecules usually composed of more than one exon. These RNAs are present in eukaryotic cells, with time and disease specificity (6). circRNAs are an increasingly important research area with huge development potential, in addition to the vast amount of studies that have investigated miRNA. Through interaction with miRNAs associated with growth, development and diseases, circRNAs can have important regulatory roles with the potential to be used a novel type of clinical diagnostic marker and as a tool to regulate cell growth and development $(28,29)$. CGRP was the first active polypeptide extracted using molecular genetics and is widely distributed in bone tissue $(30,31)$. The effect of CGRP on the function of bone tissue and bone cells has been widely investigated in previous years. According to the studies of Wang et al (31) CGRP promotes the expression of osteogenic genes and downregulates tumor necrosis factor ligand superfamily member 11 to inhibit the formation of osteoclasts, resulting in increased bone density. In previous studies, $10^{-9} \mathrm{M}$ CGRP was able to promote cell proliferation $(32,33)$. Wang et al (31) used different concentrations of 
A

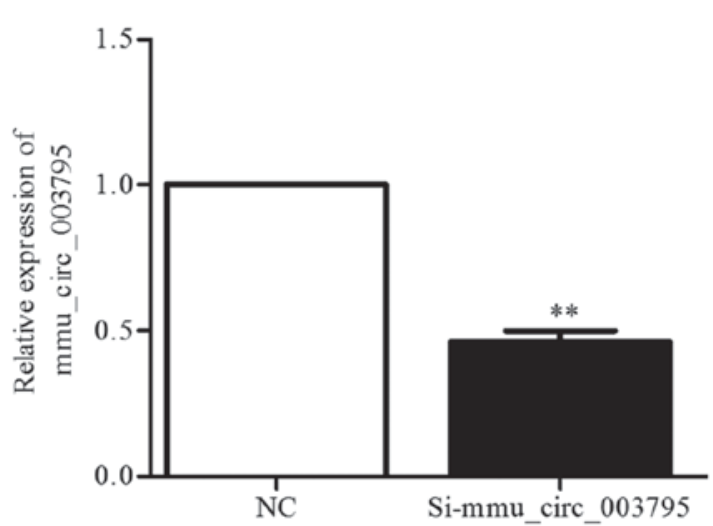

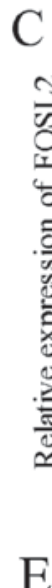

$\mathrm{E}$
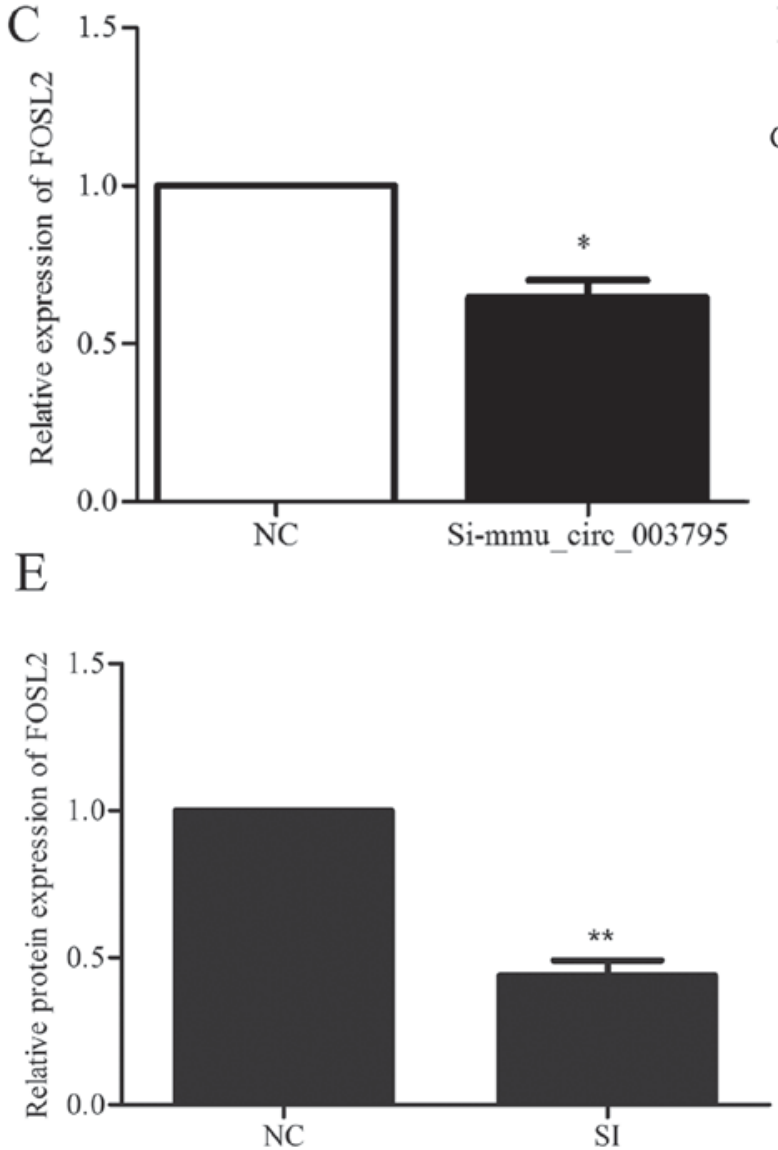

B

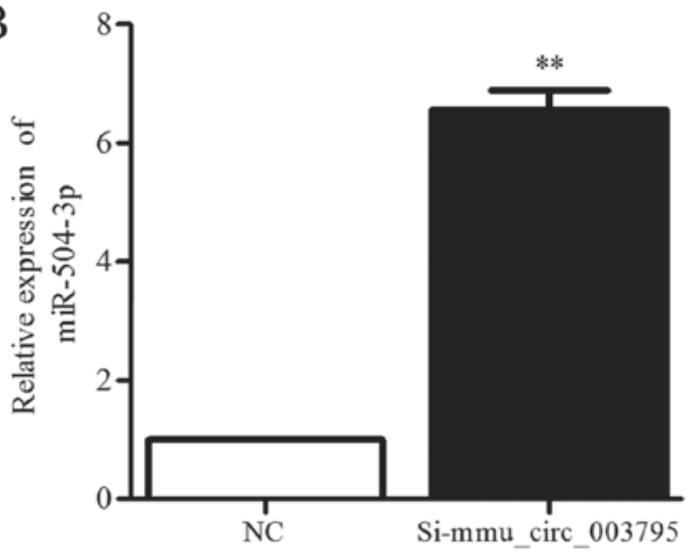

D
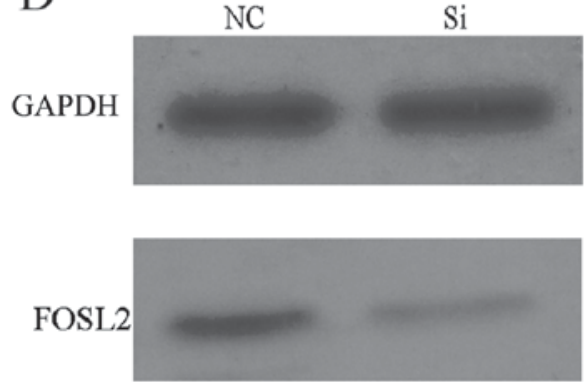

Figure 4. Changes of miR504-3p and FOSL2 expression following mmu_circRNA_003795 silencing. (A) Expression of mmu_circRNA_003795 in the interference group and negative control group analyzed by RT-qPCR $(\mathrm{P}=0.005)$. Successful transfection and knockdown, and in expression of (B) miR-504-3p $(\mathrm{P}=0.003)$ and $(\mathrm{C})$ FOSL2 $(\mathrm{P}=0.024)$ following mmu_circRNA_003795 silencing analyzed by RT-qPCR. (D) and (E) Western blot analysis of FOSL2 following mmu_circRNA_003795 silencing. ${ }^{*} \mathrm{P}<0.05$ and ${ }^{* *} \mathrm{P}<0.01$ vs. NC. RT-qPCR, reverse transcription-quantitative polymerase chain reaction; circRNA, circular RNA; miR, microRNA; FOSL2, FOS like 2 AP-1 transcription factor subunit; NC, negative control; si, small interferring.

CGRP $\left(10^{-8}, 10^{-10}\right.$ and $\left.10^{-12} \mathrm{M}\right)$ on BMSCs and the proliferation activity was tested at day 4 post-seeding. Their results indicated that $10^{-10} \mathrm{M}$ was able to promote cell proliferation. In the current study, stimulation of BMSCs with $10^{-9}$ M CGRP exerted the greatest effect on cell proliferation.

The authors' previous study also demonstrated that CGRP increases BMSC proliferation and upregulates the expression of osteogenic genes. ALP, OCN, Runx2 and OSX are essential genes required for osteogenic differentiation of BMSCs, and FOSL2 is known to have significant influence on proliferation and osteogenic differentiation of BMSCs (17). Therefore, the expression of the five genes, namely ALP, OCN, Runx2, OSX and FOSL2, in BMSCs were detected with CGRP stimulation and a blank control group. In the CGRP-treated group, FOSL2 was upregulated by 3.6-fold; ALP was upregulated by 4.3 -fold, Runx 2 was upregulated by 3.6-fold, OSX was upregulated by 2.3 -fold and $\mathrm{OCN}$ was upregulated by 3.7-fold. The results clearly demonstrated that CGRP has an important role in BMSC proliferation and differentiation. The results are similar to the previous findings (31). According to the study of Qu et al (34), circRNA microarrays are a reliable and convenient method to research circRNAs and their 
A

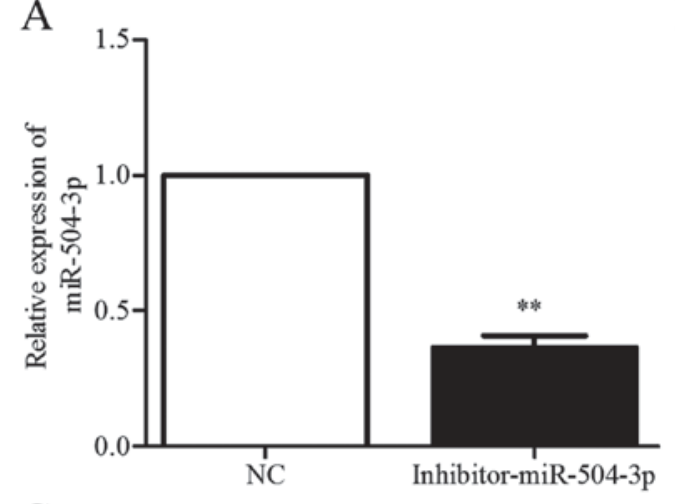

C NC Inhibitor

inhibitor

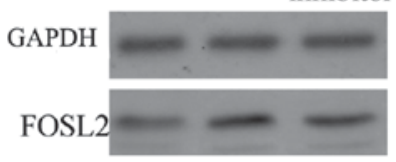

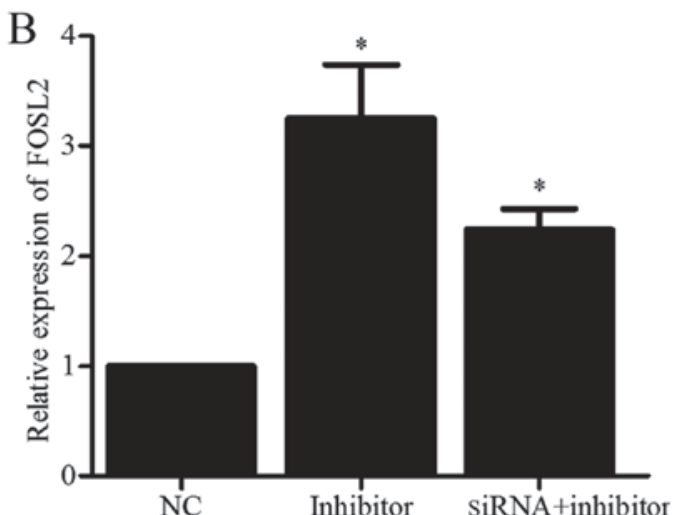

$\mathrm{D}$

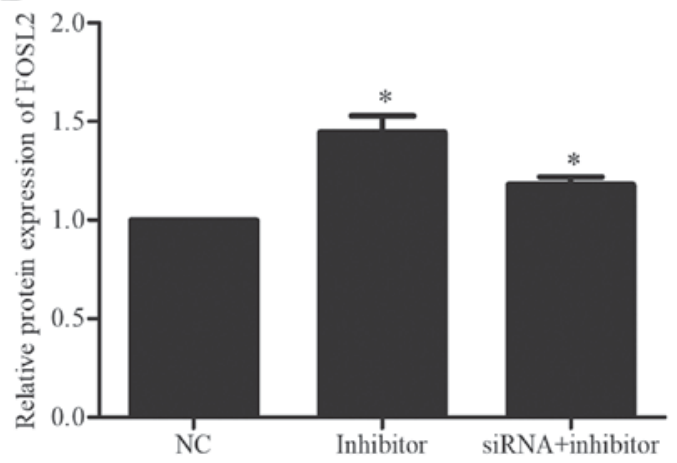

Figure 5. Effect of miR-504-3p inhibitor on FOSL2 (A) miR-504-3p inhibitor transfection was performed and the expression of miR-504-3p was detected following $72 \mathrm{~h}(\mathrm{P}=0.005)$. (B) Expression of FOSL2 following transfection with miR-504-3p inhibitor $(\mathrm{P}=0.044)$ and si-mmu_circRNA_003795 (P=0.022). (C) and (D) Western blot analysis of FOSL2 protein expression following inhibition of miR-504-3P and simultaneous inhibition of miR-504-3p and mmu circRNA_003795. "P<0.05, and ${ }^{* *} \mathrm{P}<0.01$ vs. NC. miR, microRNA; circRNA, circular RNA; si, small interfering; NC, negative control; FOSL2, FOS like 2 AP-1 transcription factor subunit.
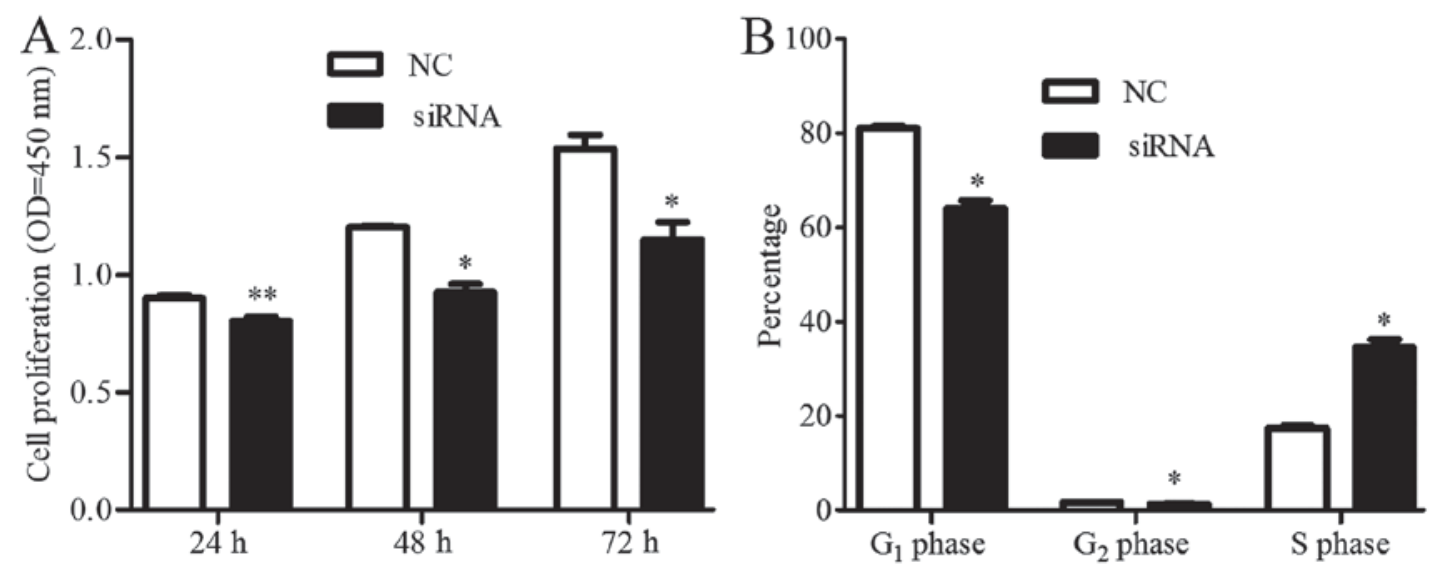

Figure 6. mmu_circRNA_003795 promotes cells proliferation and changes in the cell cycle. (A) Cell Counting Kit-8 was used to detect cell proliferation of bone marrow stem cells at 24, 48 and $72 \mathrm{~h}$ following transfection with si-mmu_circRNA_003795 (24 h, P=0.001; 48 h, P=0.014; 72 h P=0.043). (B) Flow cytometry analysis of the cell cycle in si-mmu_circRNA_003795 cells and negative control cells $(\mathrm{G} 1, \mathrm{P}=0.016 ; \mathrm{G} 2, \mathrm{P}=0.018 ; \mathrm{S}, \mathrm{P}=0.039)$. ${ }^{*} \mathrm{P}<0.05$ and ${ }^{* *} \mathrm{P}<0.01$ vs. NC. circRNA, circular RNA; NC, negative control; si, small interfering; OD, optical density.

target miRNAs and genes. Subsequently, high-throughput microarray detection of circRNAs was performed in BMSCs stimulated with CGRP. There was a total of 58 circRNAs with differential expression, of which 14 were upregulated and 44 were downregulated. Furthermore, several bioinformatics tools were used to identify miRNAs that potentially bind to the conserved seed sequence in the circRNAs and analyzed the possible target genes of these miRNAs. Subsequently, Cytoscape software was used to create a network map of the interactions between circRNAs and miRNAs. The results of the microarray analysis and the corresponding alterations in gene expression and proliferation and differentiation indicated that mmu_circRNA_003795 may have a role as an miR504-3p absorber, which results in upregulation of FOSL2 expression to ultimately promote the proliferation of BMSCs.

mmu_circRNA_003795 was differentially expressed in CGRP-treated BMSCs and the control group. The expression of mmu_circRNA_003795 in the CGRP-treated group 
was 2.9-fold compared with the blank control group, which indicated that mmu_circRNA_003795 may be associated with the proliferation of BMSCs. Electrophoresis was then used to examine the PCR product and verify the upregulation of mmu_circRNA_003795. Visualization of the DNA on the agarose gel clearly demonstrated that the size of the PCR product was the same as the expected product size and was a single band. The PCR product was sequenced and a Blast search was performed to compare the sequencing data and to establish the sequence of mmu_circRNA_003795. This also demonstrated that a circular RNA was detected, rather than a linear RNA molecule. Notably, although the expression of mmu_circRNA_003795, which was validated by RT-qPCR, exhibited the same trend in the microarray and RT-qPCR assays, the alteration in expression detected by PCR was much higher compared with the microarray. This may be due to the small sample number used for the microarray. According to the studies of Deng et al (35), the results obtained by the circRNA microarray can only be a reference method and should be confirmed by further experiments. Subsequently, an si-RNA targeting mmu_circRNA_003795 was designed to further verify its role in the proliferation of BMSCs. According to the RT-qPCR results, the expression of mmu_circRNA_003795 was inhibited by si-mmu_circRNA_003795, reduced to 0.5 -fold of the level of the negative control. This indicated that si-mmu_circRNA_003795 effectively inhibited the expression of mmu_circRNA_003795. In cells transfected with si-mmu_circRNA_003795, the expression of miR-504-3p was increased by 6.6-fold compared with the control group, which indicated that the downregulation of mmu_circRNA_003795 upregulated miR504-3p levels.

As a member of the activator factor protein 1 transcription factor family, FOSL2 is expressed in a variety of tissues. These transcription factors are associated with regulation of cell proliferation, differentiation, transformation and cell apoptosis, and with the regulation of the immune system in vivo $(36,37)$. Further study has demonstrated that FOS proteins have important roles in cell proliferation, differentiation and metabolism, growth traits, immune system responses and other physiological activities (38). FOSL2 is involved in fat metabolism, bone development and the occurrence and development of cancer, and is considered to be very important in the transcription of genes involved in osteogenic differentiation (39-42). Certain research indicates that FOSL2 exhibited an important effect on the treatment of bone loss $(42,43)$. FOSL2 is a target gene of miR-504-3p. Therefore, the expression quantity of FOSL 2 in the cells transfected with si-mmu_circRNA_003795 and a negative control was detected. The results demonstrated that the expression of FOSL2 in si-mmu_circRNA_003795 group was 0.6-fold of that of the control group. A similar result was produced by western blot analysis, indicating that mmu_circRNA_003795 indirectly regulates the expression of FOSL2.

To further verify whether miR504-3p is capable of acting on FOSL2 mRNA, an miR504-3p inhibitor was designed. The experimental results demonstrated that the expression of miR504-3p was 0.4-fold of the negative control group. Subsequently, the expression quantity of FOSL2 in the BMSCs transfected with the miR-504-3p inhibitor and the control groups were detected. The expression of FOSL 2 in the experimental group was 3.3-fold of that of the control group suggesting that miR-504-3p affects the expression of FOSL2. In conjunction with the previous conclusions, it was hypothesized that mmu_circRNA_003795 may indirectly regulate the expression of FOSL2 by acting as a sponge for miR-504-3p. In order to validate this, BMSCs were simultaneously transfected with the si-mmu_circRNA_003795 and miR-504-3p inhibitor for $72 \mathrm{~h}$, and the results demonstrated that the expression of FOSL 2 was increased by 2.2-fold. In addition, the western blot analysis demonstrated that compared with the control group, the expression of FOSL2 was increased slightly by co-transfection with si-mmu_circRNA_003795 and miR-504-3p; this was because the miR-504-3p inhibitor is more effective at inhibiting its target than si-mmu_circRNA_003795.

The effect of mmu_circRNA_003795 on the proliferation of BMSCs was also analyzed. The results demonstrated that the cell proliferation rate of BMSCs was significantly reduced by si-mmu_circRNA_003795 compared with the negative control group; the change was most significant at $72 \mathrm{~h}$ following transfection. However, the rate of change reached its peak at $48 \mathrm{~h}$, which may indicate the interference effect of the siRNA gradually weakened following 2 days in culture. Additionally, flow cytometry analysis of the cell cycle demonstrated that si-mmu_circRNA_003795 increased the proportion of cells in the G0-G1 phase and reduced the proportion of cells in the G2-M and S phase compared with the negative control group. This indicated that mmu_circRNA_003795 may affect cell proliferation by influencing the cell cycle. Notably, previous studies on FOSL2 have reported that FOSL2 mainly affects osteogenic differentiation of cells and there are few reports arguing that FOSL2 is involved in cell proliferation $(43,44)$. Therefore, it was hypothesized that FOSL 2 may promote cell osteogenesis by influencing cell proliferation; this will be investigated further in additional experiments. Furthermore, CGRP function on bone generation in vivo also should be detected and the tomography of bone following the CGRP treating will be conducted in vivo in future studies.

In conclusion, the preliminary observations of the study demonstrated that CGRP regulates miR504-3p via mmu circRNA_003795, which results in increased expression of FOSL2 and enhanced proliferation of BMSCs. However, the role of proliferation promotion during differentiation still requires further investigation.

\section{Acknowledgements}

The authors thank Dr Zhichao Zheng (Key Laboratory of Oral Medicine, Guangzhou Institute of Oral Disease, Stomatology Hospital of Guangzhou Medical University, Guangzhou, Guangdong, China) for his great help in writing this paper.

\section{Funding}

This study was supported by Science \& Technology Bureau of Guangdong Province (grant no. 2017A050501041) and Health Department of Guangdong Province (grant no. B2015089).

\section{Availability of data and materials}

The datasets used and/or analyzed during the current study are available from the corresponding author on reasonable request. 


\section{Authors' contributions}

WR, LY, TD, CW, YL, JW, ZH and FD performed the experiments and analyzed the data. LG designed the study and wrote the article. All authors approved the final manuscript.

\section{Ethics approval and consent to participate}

Not applicable.

\section{Patient consent for publication}

Not applicable.

\section{Competing interests}

The authors declare that they have no competing interests.

\section{References}

1. Jeck WR, Sorrentino JA, Wang K, Slevin MK, Burd CE, Liu J, Marzluff WF and Sharpless NE: Circular RNAs are abundant, conserved, and associated with ALU repeats. RNA 19: 141-157, 2013.

2. Sanger HL, Klotz G, Riesner D, Gross HJ and Kleinschmidt AK: Viroids are single-stranded covalently closed circular RNA molecules existing as highly base-paired rod-like structures. Proc Natl Acad Sci USA 73: 3852-3856, 1976.

3. Hsu MT and Coca-Prados M: Electron microscopic evidence for the circular form of RNA in the cytoplasm of eukaryotic cells. Nature 280: 339-340, 1979.

4. Zhang Y, Zhang XO, Chen T, Xiang JF, Yin QF, Xing YH, Zhu S, Yang $L$ and Chen LL: Circular intronic long noncoding RNAs. Mol Cell 51: 792-806, 2013.

5. Kelly S, Greenman C, Cook PR and Papantonis A: Exon skipping is correlated with exon circularization. J Mol Biol 427: 2414-2417, 2015.

6. Zhang XO, Wang HB, Zhang Y, Lu X, Chen LL and Yang L: Complementary sequence-mediated exon circularization. Cell 159: 134-147, 2014.

7. Hansen TB, Jensen TI, Clausen BH, Bramsen JB, Finsen B, Damgaard CK and Kjems J: Natural RNA circles function as efficient microRNA sponges. Nature 495: 384-388, 2013.

8. Li Z, Huang C, Bao C, Chen L, Lin M, Wang X, Zhong G, Yu B, $\mathrm{Hu}$ W, Dai L, et al: Exon-intron circular RNAs regulate transcription in the nucleus. Nat Struct Mol Biol 22: 256-264, 2015.

9. Li P, Chen S, Chen H, Mo X, Li T, Shao Y, Xiao B and Guo J: Using circular RNA as a novel type of biomarker in the screening of gastric cancer. Clin Chim Acta 444: 132-136, 2015

10. Chen S, Li T, Zhao Q, Xiao B and Guo J: Using circular RNA hsa_circ_0000190 as a new biomarker in the diagnosis of gastric cancer. Clin Chim Acta 466: 167-171, 2017

11. Zhuang ZG, Zhang JA, Luo HL, Liu GB, Lu YB, Ge NH, Zheng BY, Li RX, Chen C, Wang X, et al: The circular RNA of peripheral blood mononuclear cells: Hsa_circ_0005836 as a new diagnostic biomarker and therapeutic target of active pulmonary tuberculosis. Mol Immunol 90: 264-272, 2017.

12. Du WW, Yang W, Liu E, Yang Z, Dhaliwal P and Yang BB: Foxo3 circular RNA retards cell cycle progression via forming ternary complexes with p21 and CDK2. Nucleic Acids Res 44: 2846-2858, 2016.

13. Xia W, Qiu M, Chen R, Wang S, Leng X, Wang J, Xu Y, Hu J, Dong G, Xu PL and Yin R: Circular RNA has_circ_0067934 is upregulated in esophageal squamous cell carcinoma and promoted proliferation. Sci Rep 6: 35576, 2016.

14. Liu Q, Zhang X, Hu X, Dai L, Fu X, Zhang J and Ao Y: Circular RNA related to the chondrocyte ECM regulates MMP13 expression by functioning as a MiR-136 'Sponge' in human cartilage degradation. Sci Rep 6: 22572, 2016.

15. Chen L, Zhang S, Wu J, Cui J, Zhong L, Zeng L and Ge S: circRNA_100290 plays a role in oral cancer by functioning as a sponge of the miR-29 family. Oncogene 36: 4551-4561, 2017.
16. Liu H, Peng H, Wu Y, Zhang C, Cai Y, Xu G, Li Q, Chen X, Ji J, Zhang Y and OuYang HW: The promotion of bone regeneration by nanofibrous hydroxyapatite/chitosan scaffolds by effects on integrin-BMP/Smad signaling pathway in BMSCs. Biomaterials 34: 4404-4417, 2013.

17. Hu N, Feng C, Jiang Y, Miao Q and Liu H: Regulative effect of Mir-205 on osteogenic differentiation of bone mesenchymal stem cells (BMSCs): Possible role of SATB2/Runx2 and ERK/MAPK pathway. Int J Mol Sci 16: 10491-10506, 2015.

18. Özdal-Kurt F, Tuğlu I, Vatansever HS, Tong S and Deliloğlu-Gürhan SI: The effect of autologous bone marrow stromal cells differentiated on scaffolds for canine tibial bone reconstruction. Biotech Histochem 90: 516-528, 2015.

19. Novikova LN, Brohlin M, Kingham PJ, Novikov LN and Wiberg M: Neuroprotective and growth-promoting effects of bone marrow stromal cells after cervical spinal cord injury in adult rats. Cytotherapy 13: 873-887, 2011.

20. Russell FA, King R, Smillie SJ, Kodji X and Brain SD: Calcitonin gene-related peptide: Physiology and pathophysiology. Physiol Rev 94: 1099-1142, 2014.

21. Liang W, Zhuo X, Tang Z, Wei X and Li B: Calcitonin gene-related peptide stimulates proliferation and osteogenic differentiation of osteoporotic rat-derived bone mesenchymal stem cells. Mol Cell Biochem 402: 101-110, 2015.

22. Takahashi N, Matsuda Y, Sato K, de Jong PR, Bertin S, Tabeta K and Yamazaki K: Neuronal TRPV1 activation regulates alveolar bone resorption by suppressing osteoclastogenesis via CGRP. Sci Rep 6: 29294, 2016.

23. He H, Chai J, Zhang S, Ding L, Yan P, Du W and Yang Z: CGRP may regulate bone metabolism through stimulating osteoblast differentiation and inhibiting osteoclast formation. Mol Med Rep 13: 3977-3984, 2016.

24. Liang W, Li L, Cui X, Tang Z, Wei X, Pan H and Li B: Enhanced proliferation and differentiation effects of a CGRP- and Sr-enriched calcium phosphate cement on bone mesenchymal stem cells. J Appl Biomater Funct Mater 14: e431-e440, 2016.

25. R Core Team: R: A language and environment for statistical computing. version 3.1.2. R foundation for statistical computing, Vienna, 2014. http://www.R-project.org/

26. Livak KJ and Schmittgen TD: Analysis of relative gene expression data using real-time quantitative PCR and the 2(-Delta Delta $\mathrm{C}(\mathrm{T}))$ method. Methods 25: 402-408, 2001

27. Wu C, Zheng Z, Ren W, Deng T, Li Y, Yang L, Wu J, Huang Z, Li Z and Guo L: Mm9_circ_009056 enhances osteogenesis by targeting BMP7 via CGRP-mediated miR-22-3p. Biochem Biophys Res Commun 501: 199-205, 2018.

28. Memczak S, Jens M, Elefsinioti A, Torti F, Krueger J, Rybak A, Maier L, Mackowiak SD, Gregersen LH, Munschauer M, et al: Circular RNAs are a large class of animal RNAs with regulatory potency. Nature 495: 333-338, 2014.

29. Peng Y, Song X, Zheng Y, Wang X and Lai W: Circular RNA profiling reveals that circCOL3A1-859267 regulate type I collagen expression in photoaged human dermal fibroblasts. Biochem Biophys Res Commun 486: 277-284, 2017.

30. Sample SJ, Hao Z, Wilson AP and Muir P: Role of calcitonin gene-related peptide in bone repair after cyclic fatigue loading. PLoS One 6: e20386, 2011.

31. Wang L, Shi X, Zhao R, Halloran BP, Clark DJ, Jacobs CR and Kingery WS: Calcitonin-gene-related peptide stimulates stromal cell osteogenic differentiation and inhibits RANKL induced NF-kappaB activation, osteoclastogenesis and bone resorption. Bone 46: 1369-1379, 2010.

32. Cornish J, Callon KE, Bava U, Kamona SA, Cooper GJ and Reid IR: Effects of calcitonin, amylin, and calcitonin gene-related peptide on osteoclast development. Bone 29: 162-168, 2001.

33. Li Y, Yang L, Zheng Z, Li Z, Deng T, Ren W, Wu C and Guo L: Bio-Oss ${ }^{\circledR}$ modified by calcitonin gene-related peptide promotes osteogenesis in vitro. Exp Ther Med 14: 4001-4008, 2017.

34. Qu S, Song W, Yang X, Wang J, Zhang R, Zhang Z, Zhang H and Li H: Microarray expression profile of circular RNAs in human pancreatic ductal adenocarcinoma. Genom Data 5: 385-387, 2015.

35. Deng T, Yang L, Zheng Z, Li Y, Ren W, Wu C and Guo L: Calcitonin gene-related peptide induces IL-6 expression in RAW264.7 macrophages mediated by mmu_circRNA_007893. Mol Med Rep 16: 9367-9374, 2017.

36. Weekes D, Kashima TG, Zandueta C, Perurena N, Thomas DP, Sunters A, Vuillier C, Bozec A, El-Emir E, Miletich I, et al: Regulation of osteosarcoma cell lung metastasis by the c-Fos/AP-1 target FGFR1. Oncogene 35: 2852-2861, 2016. 
37. Kushibiki T, Tu Y, Abu-Yousif AO and Hasan T: Photodynamic activation as a molecular switch to promote osteoblast cell differentiation via AP-1 activation. Sci Rep 5: 13114, 2015.

38. Jahangiri L, Sharpe M, Novikov N, González-Rosa JM, Borikova A, Nevis K, Paffett-Lugassy N, Zhao L, Adams M, Guner-Ataman B, et al: The AP-1 transcription factor component Fosl2 potentiates the rate of myocardial differentiation from the zebrafish second heart field. Development 143: $113-122,2016$

39. Wang J, Sun D, Wang Y, Ren F, Pang S, Wang D and Xu S: FOSL2 positively regulates TGF- $\beta 1$ signalling in non-small cell lung cancer. PLoS One 9: e112150, 2014.

40. Luther J, Ubieta K, Hannemann N, Jimenez M, Garcia M, Zech C, Schett G, Wagner EF and Bozec A: Fra-2/AP-1 controls adipocyte differentiation and survival by regulating PPAR $\gamma$ and hypoxia. Cell Death Differ 21: 655-664, 2014.

41. Hasenfuss SC, Bakiri L, Thomsen MK, Hamacher R and Wagner EF: Activator protein 1 transcription factor Fos-related antigen 1 (Fra-1) is dispensable for murine liver fibrosis, but modulates xenobiotic metabolism. Hepatology 59: 261-273, 2014.
42. Li J, Li S, Hu Y, Cao G, Wang S, Rai P, Wang X and Sun K: The expression level of mRNA, protein, and DNA methylation status of FOSL2 of Uyghur in XinJiang in type 2 diabetes. J Diabetes Res 2016: 1-7, 2016.

43. Bozec A, Bakiri L, Jimenez M, Schinke T, Amling M and Wagner EF: Fra-2/AP-1 controls bone formation by regulating osteoblast differentiation and collagen production. J Cell Biol 190: 1093-1106, 2010.

44. Bozec A, Bakiri L, Jimenez M, Rosen ED, Catalá-Lehnen P, Schinke $T$, Schett $G$, Amling $M$ and Wagner EF: Osteoblast-specific expression of Fra-2/AP-1 controls adiponectin and osteocalcin expression and affects metabolism. J Cell Sci 126: 5432-5440, 2013.

This work is licensed under a Creative Commons Attribution-NonCommercial-NoDerivatives 4.0 International (CC BY-NC-ND 4.0) License. 